\title{
Efficacy of MTAD Solution and Er:YAG Laser in Smear Layer Removal from Extracted Root Canals: A SEM Evaluation
}

\author{
Isıl Ozgul Kalyoncu ${ }^{1}$ (D), Figen Eren Giray ${ }^{1}$ (D), Basak Durmus ${ }^{1}$ (D), Yildiz Garip Berker ${ }^{2}$ (D), Ilknur Tanboga ${ }^{1}$ (D) \\ ${ }^{1}$ Department of Pediatric Dentistry, Marmara University School of Dentistry, Istanbul, Turkey. \\ ${ }^{2}$ Department of Endodontics, Kent University School of Dentistry, Istanbul, Turkey.
}

Correspondence Author: Işıl Ozgul Kalyoncu

E-mail: ikalyoncu@marmara.edu.tr

Received: $14.09 .2020 \quad$ Accepted: 14.12 .2020

\begin{abstract}
Objective: The aim of this study was to compare the efficacy of MTAD (a mixture of a tetracycline isomer, an acid, and a detergent), Er:YAG laser, $17 \% \mathrm{EDTA}$, and $5.25 \% \mathrm{NaOCl}$ in removing the smear layer from the surface of instrumented root canals.

Methods: Various organic acids, instruments and lasers have been used to remove the smear layer from the surface of instrumented root canals. Twenty-eight extracted maxillary and mandibular permanent incisors were prepared with rotary files. The teeth were randomly allocated to four treatment groups for final irrigation as follows: (1) 17\% EDTA (followed by NaOCl), (2) 5.25\% NaOCl, (3) Er:YAG laser, and (4) MTAD. All teeth were processed for scanning electron microscopy (SEM) and the removal of the smear layer was examined in the apical, middle and coronal thirds.

Results: At coronal location, $\mathrm{NaOCI}(2.2 \pm 0.4)$ group had significantly higher scores than MTAD(0.0 \pm 0.0$), E D T A(0.6 \pm 0.4)$ and Er:YAG laser(0.6 \pm 0.4$)$ groups $(p<0.001, p=0.039$, and $p=0.039$, respectively). At the middle third, $\mathrm{NaOCl}(2.6 \pm 0.5)$ scores were significantly higher than $\mathrm{MTAD}(0.0 \pm 0.0)$ and EDTA $(0.8 \pm 0.4)$ groups ( $<<0.001$ and $p=0.036$ respectively). At apical MTAD $(0.4 \pm 0.3)$ group had significantly better scores $(p<0.001)$.

Conclusion: The results of this suggest that MTAD is an effective final irrigator agent, particularly in the apical segment of the root canal, which presents challenges during cleaning.

Keywords: MTAD, Er:YAG laser, smear layer, scanning electron microscope
\end{abstract}

\section{INTRODUCTION}

The long-term survival of endodontic treatment is an issue of high priority focus in pediatric dentistry. The success of endodontic treatment depends on a number of factors including a good biomechanical shaping, disinfection, and three-dimensional filling of the root canal system (1-3). The mechanical instrumentation utilized for the purpose of cleaning and shaping the root canal system results in the formation of smear layer covering the walls of dentin that not only contains dentin residues, but also the remnants of odontoblastic processes, pulp remnants, as well as bacteria $(1,4)$. Therefore, canal treatment should not consist only of removing the pulp and forming the root canal, we must focus on the complete removal of the smear layer. Its removal provides more efficient penetration of canal sealers and adhesion to the dentin walls $(1,2,4)$. Structural irregularities of the root canal system do not allow complete removal of the debridement by mechanical instrumentation.

A number of different procedures including chemical, ultrasonic, and laser-assisted methods have been tested for removing the smear layer. At the same time, numerous irrigation agents with specific chelation action, dissolving capacity, and antibacterial properties have been introduced over the years (5). Recently, compounds combining acids, detergents, and antibiotics have been the focus of attention as a means of reducing the surface tension as well as for providing better penetration to dentin tubules (6).

For many years, $\mathrm{NaOCl}$ (sodium hypochlorite) has been conventionally used as a canal irrigation agent due to its antibacterial properties (7). Although $\mathrm{NaOCl}$ is effective in removing the organic constituents of the smear layer, chelating agents such as citric acid, polyacrylic acid, tannic acid, phosphoric acid or ethylenediamine tetra-acetic acid (EDTA) are required for the removal of inorganic material(8). Originally, 17\% EDTA was used to demineralize the root canal dentin in narrowed and sclerosed canals (6). Dental laser Er:YAG (erbium:yttrium-aluminum-garnet) showed that it can remove most of the smear layer in the root canal wall and open dentin tubules (9). The use of these methods has 
been tested and the smear layer has not been able to remove the entire length of the channel (3).

MTAD, which is a tetracycline isomer, is a bio-compatible material consisting of a mixture of acid and detergent. It exerts a dissolving effect on the pulp and dentin similar to that of EDTA. Torabinejad et al.(10) reported that MTAD is able to effectively remove the smear layer, to eliminate micro-organisms resistant to previous agents, and to provide long-lasting anti-microbial activity.

Therefore, this in vitro study was designed to compare the efficacy of MTAD (a mixture of tetracycline isomer, acid and detergent) for the removal of intra-canal smear with that of $5.25 \% \mathrm{NaOCl}, 17 \%$ EDTA, and Er:YAG laser using scanning electron microscope (SEM) images.

\section{METHODS}

Our study is an in-vitro study performed on teeth extracted due to different extraction indications and all stages of our study were conducted in accordance with the World Medical Association Declaration of Helsinki, IKU/ILU criteria. Twentyeight maxillary and mandibular single rooted permanent teeth having mature root apex and single root canal with similar anatomic characteristics without anatomical variations and root resorption were selected and included in the study. These extracted teeth were stored in \%1 thymol solution. Teeth were decoronated from the cementoenamel junction with a low-speed rotary diamond disk (90 $\mu \mathrm{m}$; Microdont, Brazil) under coolant water. The root length of teeth was measured using \#10 K-file (Mani ${ }^{\circledR}$, Mani Inc., Japan). The root canal length measurements were performed in such a manner that the tip of the canal equipment could be visible at the apex of the root canal, and $1 \mathrm{~mm}$ was subtracted from the reading to obtain the measurement length to be used for study analyses. The shaping of the root canals was performed with crown-down technique and ProTaper Universal (Dentsply Maillefer, Ballaigues, Switzerland) rotary files (S1 through F3). Each file was used for preparing only for four root canals. Following each file use, root canals were irrigated with $3 \mathrm{ml} 5.25 \% \mathrm{NaOCl}$. After a final irrigation with $3 \mathrm{ml}$ of $5.25 \% \mathrm{NaOCl}$, rinsing with $3 \mathrm{ml}$ of distilled water was carried out to prevent the formation of $\mathrm{NaCl}$ crystals, and the canals were dried with absorbent paper points. After shaping the canals, the apical foramina of all canals were sealed with modeling compound to prevent outflow of the irrigation solution. The teeth were randomly allocated to four treatment groups for the removal of smear layer: (1) 17\% EDTA (followed by $\mathrm{NaOCl}$ ), (2) $5.25 \% \mathrm{NaOCl}$, (3) Er:YAG laser (Fotona Medical Lasers, Fidelis PLUS, Er:YAG and Nd:YAG Dental Laser, Slovenia) and (4) MTAD (MTAD Biopure, Tulsa, OK, USA). The final irrigation protocol in each group determined in Table-1.

During Er:YAG procedure, fiber tip of the laser applicator was positioned within the canal as to lie along the length of the canal and parallel to the surface. Procedure involved activation of the laser equipment at apex and small rotation-like movements in apical-coronal direction for 15 seconds while taking care to provide contact between the fiber tip and whole surface of the root canal. The procedure was repeated for 4 times, with 15 second intervals to prevent excessive heat formation.

In order to ensure that no test solution has remained, all study specimens were washed out with $3 \mathrm{ml}$ of distilled water and cotton pellets were used to seal the coronal tip of the root canals for preventing the entry of foreign material into the canal. Two longitudinal grooves were prepared on the buccal and lingual aspects of each root using diamond bur without penetration into the canal. The roots were then split into two halves with chisel. For each root, the half containing the most visible part of the apex was conserved and coded, the other part was discarded. The specimens were kept overnight in a desiccator at $60^{\circ} \mathrm{C}$. The coded specimens were then mounted on metallic stubs, covered with 0.02-micron thick $\left(226.8 \mathrm{~A}^{\circ}\right)$ gold and examined on scanning electron microscope (SEM, JEOL JSM-5910, Tokyo, Japan). Serial (SEM) photomicrographs at $X 1000$ magnification were taken at the coronal, middle and apical thirds of the root canals. The photographs were evaluated for the presence of a smear layer. SEM images obtained were assessed using the scoring system proposed by Takeda et al. and modified by Prado et al.(11). A score between 0 and 3 was assigned to each photomicrograph based on the presence of debris and smear layer as well as the patency of the dentin tubules. Scoring system was as follows: 0, no smear layer and debris at all, with all tubules cleaned and opened; 1 , a few areas covered by smear layer and debris, with most tubules cleaned and opened; 2, smear layer and debris covering almost all surface, with few tubules opened; 3, smear layer and debris covering all the surfaces. Scoring was done in a blinded manner by two dentists who were not informed on the nature and purpose of these experiments and the average scores were used for the analyses. The kappa values for inter-examiner agreement were $0.730,0.949$, and 0.801 for coronal, middle and apical measurements, respectively.

\subsection{Statistical analysis}

For the analysis of data, SPSS (Statistical Package for Social Sciences) version 23 was used.

One-way anova test was used for intergroup comparisons of data and built-in post hoc Bonferroni test was used for pairwise comparisons. For inter-examiner agreement, Kappa values were calculated. A p value smaller than 0.05 was used as an indication of statistical significance.

\section{RESULTS}

Figure 1 show the representative images of the apical, middle, and coronal thirds of root dentin covered by smear layer various irrigation protocols of 4 groups. Group 1 (17\% EDTA + 5.25\% NaOCL): In the coronal third of the samples, a few areas covered by smear layer more intense smear layer was observed in the middle triple. In apical one third, smear 
layer covering almost all surface, with few tubules opened. Group 2 (5.25\% NaOCL): In all parts of the root of the tooth, a dense smear layer was observed in a third of the coronal, middle and apical. Group 3 (Er:YAG laser): In the coronal third of the samples, a few areas covered by smear layer and in the middle and apical third smear layer covering almost all surface, with few tubules opened. Group 4 (MTAD): No smear layer was observed in the coronal and middle third of all samples. The smear layer was not observed in the apical third of the 2 samples, in 5 samples of 7, small amount of smear layer was observed in the apical third part of the root canal.

When the data obtained from SEM images were examined, MTAD group from all groups showed that the best result was obtained in the coronal, middle and apical third Scores differed significantly across groups at all locations (coronal, middle and apical) $p<0.001$. Table 2 shows comparisons of the scores between study groups. At coronal location, $\mathrm{NaOCl}$ group had significantly higher scores than in MTAD, EDTA and Er:YAG laser groups $(p<0.001, p=0.039$, and $p=0.039$, respectively). At the middle third, $\mathrm{NaOCl}$ scores were significantly higher than MTAD and EDTA groups $(p<0.001$ and $p=0.036$ respectively). At apical MTAD $(0.4 \pm 0.3)$ group had significantly better scores $(p<0.001)$.

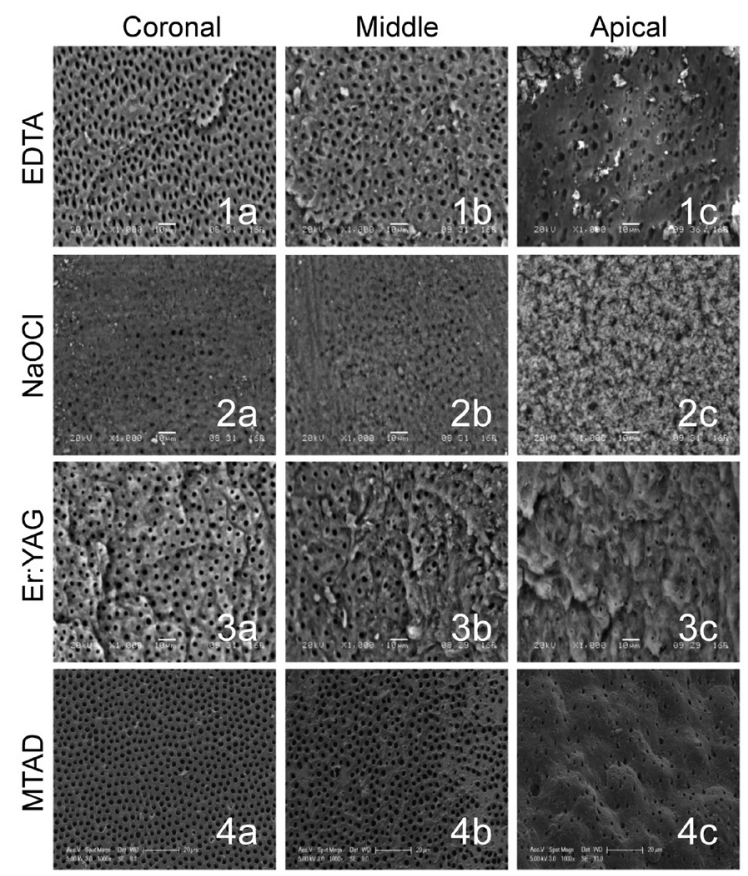

Figure 1. Exemplary scanning electron microscope (SEM) images. In the coronal (1a) and middle (1b) thirds of the root canal in Group 1 (17\% EDTA), the dentin tubules are clearly observed, while smear remnants together with open dentin tubules can be seen in the apical third (1c). In Group 2 (5.25\% NaOCl), the smear layer covering the dentin tubules in all root surface are seen $(2 a, 2 b, 2 c)$. The open dentin tubules of the root canal in the coronal (3a) and middle ( $3 b$ ) thirds are seen in a specimen from Group 3 (Er:YAG laser), while the apical (3c) segment displays smear remnants together with open dentin tubules. In group 4, MTAD $(4 a, 4 b)$ No smear layer was observed in the coronal and middle third. small amount of smear layer was observed in the apical third (4c).
Table 1. Final irrigation protocols of the study groups.

\begin{tabular}{|l|l|l|}
\hline Group & $\begin{array}{l}\text { Final solution for } \\
\text { removal of the smear } \\
\text { layer }\end{array}$ & Application procedure \\
\hline Group 1 $(n=7)$ & $17 \%$ EDTA & $\begin{array}{l}1 \mathrm{~min} 5 \mathrm{ml} \mathrm{17 \%} \mathrm{EDTA} \mathrm{plus} \mathrm{3ml} \\
5.25 \% \mathrm{NaOCl}\end{array}$ \\
\hline Group 2 $(\mathrm{n}=7)$ & $5.25 \% \mathrm{NaOCl}$ & $1 \mathrm{~min} 5 \mathrm{ml}$ \\
\hline Group 3 $(\mathrm{n}=7)$ & Er:YAG laser & $\begin{array}{l}\text { Short pulse mode, optical fiber } \\
\text { diameter 0.3mm, wavelength } \\
2940 \mathrm{~nm} \text { output power 1W, } \\
\text { pulse energy 100mJ, pulse } \\
\text { frequency 10Hz. }\end{array}$ \\
\hline Group 4 (n=7) & MTAD & $\begin{array}{l}\text { Prepared freshly by mixing the } \\
\text { powder Part A and liquid Part } \\
\text { B; agitated with a no:15 k-file } \\
\text { using 4ml solution for 5min }\end{array}$ \\
\hline
\end{tabular}

EDTA, ethylenediamine tetraacetic acid; $\mathrm{NaOCl}$, sodium hypochloride; Er:YAG, erbium yttrium aluminium garnet; MTAD, a mixture of a tetracycline isomer, an acid, and a detergent

Table 2. Comparisions of the scores between study groups

\begin{tabular}{lccccc} 
& $\begin{array}{c}\text { EDTA } \\
(\mathbf{n}=\mathbf{7})\end{array}$ & $\begin{array}{r}\mathbf{N a O C l} \\
(\mathbf{n}=\mathbf{7})\end{array}$ & $\begin{array}{c}\text { Er:YAG laser } \\
(\mathbf{n}=\mathbf{7})\end{array}$ & $\begin{array}{c}\text { MTAD } \\
(\mathbf{n}=\mathbf{7})\end{array}$ & $\mathbf{p}^{*}$ \\
\hline Coronal & $0.6 \pm 0.4$ & $2.2 \pm 0.4$ & $0.6 \pm 0.4$ & $0.0 \pm 0.0$ & $<0.001$ \\
\hline Middle & $0.8 \pm 0.4$ & $2.6 \pm 0.5$ & $1.0 \pm 0.6$ & $0.0 \pm 0.0$ & $<0.001$ \\
\hline Apical & $1.4 \pm 0.5$ & $3.0 \pm 0.0$ & $1.4 \pm 0.5$ & $0.4 \pm 0.3$ & $<0.001$ \\
\hline
\end{tabular}

Data presented as mean \pm standard deviation. * $p$ for overall difference

\section{DISCUSSIONS}

In this study comparing the efficacy of three different irrigation agents and Er:YAG laser in removing the smear layer formed after mechanical shaping of the root canal, MTAD showed superiority over $\mathrm{NaOCl}$ in all parts of the root canal, while Er:YAG laser and 17\% EDTA were more effective than $\mathrm{NaOCl}$ in the coronal and middle third of the root, and $\mathrm{NaOCl}$ had the worst performance statistically in all root sections with regard to smear scores. Despite numerous previous comparisons between MTAD and different irrigation agents in terms of their ability to remove the smear layer from the root canal, studies comparing this agent with laser are scarce in number $(1,12-14)$.

Scanning electron microscopy (SEM) was used in this study to assess the smear layer as an effective means to examine the morphological changes occurring on the surface of the root canal surface. In SEM images, smear layer has an appearance that is akin to remnants of mug (1). The diameter and density of the dentin tubules exhibit variations along the root canal from coronal to apical segments, with dentin tubules showing irregular frequency and angles in the apical segment. The apical third of the root canal represents the most challenging segment for cleaning as the size of the canal is significantly reduced (15). Due to these anatomical variations, the root canal was evaluated in three segments as apical, middle and coronal parts.

An ideal canal irrigation agent should have antibacterial properties, be able to dissolve the organic and inorganic 
material without causing erosions in the dentin, remove the smear layer, have no toxic effects on periapical tissues, be bio-compatible, and should be able to dissolve and remove the debridement in the root canal system $(8,15,16)$.

$\mathrm{NaOCl}$ with concentrations ranging between 0.5 to $5.25 \%$ is the most widely used agent for root canal treatments, based on its antimicrobial properties as well dissolving capacity (17). However, its efficacy in the removal of the smear layer is limited. In the study by Andrabi et al. (18) examining the efficacy of a number of different irrigation agents in removing the intra-canal smear layer, $3 \% \mathrm{NaOCl}$ was no more effective than distilled water and the authors concluded that $\mathrm{NaOCl}$ was ineffective for this purpose when used alone (18). Similarly, Gupta et al. found that $\mathrm{NaOCl}$ had an effect only on the organic tissue, with no efficacy in the removal of the smear layer (1). In line with these previous observations (3, 12 ), in this study, $\mathrm{NaOCl}$ solution failed to clear the smear layer in the root canal.

The amount of the material used and duration of administration have been reported to influence the smearremoving capacity of different irrigation agents, and these agents have been generally found to be more effective in the coronal and middle thirds of the canal as compared to the apical third, due to wider root canals in the coronal and middle segments than in the apical segment allowing a more extensive contact between the solutions and root surface (15). It has been reported by Torabinejad et al. (10) found that MTAD, when used as a final irrigating agent together with $\mathrm{NaOCl}$, can effectively remove the smear layer without altering the structure of the dentin tubules. MTAD is an acidic solution with a $\mathrm{pH}$ of 2.15 that can also eliminate the inorganic substances, (10) and in this study, it was effective in the most challenging apical third of the root canal similar to the reports by Gupta et al., Andrabi et al., Poul et al., and Kumar et al. (1, 3, 4, 13, 18).

A clean root canal surface was achieved by the combination of $17 \%$ EDTA and $5.25 \% \mathrm{NaCl}$ in the study by Yamada et al.(19). Currently, sequential administration of $17 \%$ EDTA and $5.25 \% \mathrm{NaOCl}$ irrigation solutions represents a widely accepted practice for the elimination of organic and inorganic constituents of the smear layer, (1) although this formulation may fail to eradicate the smear layer completely in the apical segment, despite complete removal in the coronal and middle thirds(3). Torabineajad et al.(10) found similar efficacy between 17\% EDTA and MTAD in the coronal and middle segments, while MTAD was more effective in the apical part; furthermore, 17\% EDTA was associated with erosion of root surface dentin (11). Despite the success of $17 \%$ EDTA in the removal of the smear layer, this solution has also been reported to lead to erosions in the peri-tubular and inter-tubular dentin in the coronal and middle segments of the root canal as well as in open dentin tubules $(1,11,13$, 18).

In the study by Kalyoncuoglu and Demiryurek, (9) assessing a number of different irrigation agents and methods for the removal of smear layer (Er:YAG, Nd:YAG, $5.25 \% \mathrm{NaOCl}$,
17\%EDTA, MTAD), combination of $17 \%$ EDTA and 5.25 $\mathrm{NaOCl}$ was the most effective means for smear removal and the authors underscored the fact that this efficacy was associated with allowing a 5 minute treatment period for $17 \%$ EDTA solution within the root canal. On the other hand, in a study by Teixeria et al. where SEM was used to compare different durations of treatment with 17\% EDTA and 5.25\% $\mathrm{NaOCl}$ with respect to smear removal in the root canal, treatment durations of 1,3 , or 5 minutes were found to have comparable efficacy (7). In the light of this latter piece of information, a 1-minute treatment duration was chosen for $17 \%$ EDTA application in our study in order to minimize the erosive effect. Significant differences between $17 \%$ EDTA $/ 5.25 \% \mathrm{NAOCl}$ combination and other methods were observed in the removal of the smear layer in the apical third of the canal except MTAD in the current study. These were parallel with the report by Charlie et al.(3) but in contrast with the report by Kalyoncuoglu and Demiryurek (9).

Laser has also been used within the root canal for the eradication of organisms and removal of the smear layer. Ashraf et al. (20) reported higher efficacy for $17 \%$ EDTA than Er:YAG laser in their SEM study which comparing two irrigation solutions and laser treatment in the apical third. Kalyoncuoglu and Demiryurek, (9) failed to observe a smear removal effect for Er:YAG or Nd:YAG laser methods. Guidotti et al.(5) evaluated Er:YAG laser in combination with $\mathrm{NaOCl}$ and EDTA with regard to smear removal capacity and found that combination of laser with EDTA was statistically superior in all root canal surfaces as compared to treatment with laser or EDTA alone. In that same study, laser alone was the least effective approach. In contrast, Er:YAG laser was significantly more effective than $\mathrm{NaOCl}$ solution in the coronal third of the canal in our study, despite the absence of a difference from other irrigation agents in the middle and apical thirds. These observed differences may have resulted from a number of factors such as the strength of the laser beam used, the amount of light absorbed by the tissues and duration of exposure, differences in the tips and tip-to target distances, and also from other methodological discrepancies.

\section{CONCLUSIONS}

Although our findings may be considered preliminary due to limited sample size, SEM results suggest that MTAD, laser and EDTA exhibit comparable efficacy in smear removal in the middle and coronal third of the root canal, while MTAD may distinguish itself from other methods as an effective irrigation agent in the apical third of the root canal. This is notable considering the difficulty apex presents when cleaning the root canal.

\section{Conflict of Interest}

There are no conflicts of interest in connection with this paper. 


\section{REFERENCES}

[1] Gupta PK, Mahajan UP, Gupta K, Sheela NV. Comparative evaluation of a new endodontic irrigant - mixture of a tetracycline isomer, an acid, and a detergent to remove the intracanal smear layer: a scanning electron microscopic study. J Int Oral Health 2015;7:1-6.

[2] Susila A, Minu J. Activated Irrigation vs. Conventional nonactivated irrigation in endodontics - A systematic review. Eur Endod J 2019;4:96-110.

[3] Charlie KM, Kuttappa MA, George L, Manoj KV, Joseph B, John NK. A Scanning electron microscope evaluation of smear layer removal and antimicrobial action of mixture of tetracycline, acid and detergent, sodium hypochlorite, ethylenediaminetetraacetic acid, and chlorhexidine gluconate: An in vitro study. J Int Soc Prev Community Dent 2018;8:62-9.

[4] Kumar P, Prasad N, Darawade A, Bhagat SK, Narayana N, Darawade P. The Effect of four commonly used root canal irrigants on the removal of smear layer: An in-vitro scanning electron microscope study. J Int Oral Health 2015;7:88-93.

[5] Guidotti R, Merigo E, Fornaini C, Rocca JP, Medioni E, Vescovi P. Er:YAG 2,940-nm laser fiber in endodontic treatment: a help in removing smear layer. Lasers Med Sci 2014;29:69-75.

[6] Karunakaran JV, Kumar SS, Kumar M, Chandrasekhar S, Namitha D. The effects of various irrigating solutions on intraradicular dentinal surface: An SEM analysis. J Pharm Bioallied Sci 2012;4:S125-30.

[7] Teixeira CS, Felippe MC, Felippe WT. The effect of application time of EDTA and $\mathrm{NaOCl}$ on intracanal smear layer removal: an SEM analysis. Int Endod J 2005;38:285-90.

[8] Dotto L, Sarkis Onofre R, Bacchi A, Rocha Pereira GK. Effect of root canal irrigants on the mechanical properties of endodontically treated teeth: A scoping review. J Endod 2020;46:596-604.e3.

[9] Kalyoncuoglu E, Demiryurek EO. A comparative scanning electron microscopy evaluation of smear layer removal from teeth with different irrigation solutions and lasers. Microsc Microanal 2013;19:1465-9.
[10] Torabinejad M, Khademi AA, Babagoli J, Cho Y, Johnson WB, Bozhilov K. A new solution for the removal of the smear layer. J Endod 2003;29:170-5.

[11] Zhou H, Li Q, Wei L, Huang S, Zhao S. A comparative scanning electron microscopy evaluation of smear layer removal with chitosan and MTAD. Niger J Clin Pract 2018;21:76-80.

[12] Yadav HK, Yadav RK, Chandra A, Tikku AP. A Scanning electron microscopic evaluation of the effectiveness of etidronic acid, SmearClear and MTAD in removing the intracanal smear layer. J Dent (Shiraz) 2017;18:118-26.

[13] Paul ML, Mazumdar D, Niyogi A, Baranwal AK. Comparative evaluation of the efficacy of different irrigants including MTAD under SEM. J Conserv Dent 2013;16:336-41.

[14] Srikumar GP, Sekhar KS, Nischith KG. Mixture tetracycline citric acid and detergent - A root canal irrigant. A review. J Oral Biol Craniofac Res 2013;3:31-5.

[15] Mohammadi Z, Shalavi S, Yaripour S, Kinoshita JI, Manabe A, Kobayashi M. Smear layer removing ability of root canal irrigation solutions: A review. J Contemp Dent Pract 2019;20:395-402.

[16] Deari S, Mohn D, Zehnder M. Dentine decalcification and smear layer removal by different ethylenediaminetetraacetic acid and 1-hydroxyethane-1,1-diphosphonic acid species. Int Endod J 2019;52:237-243.

[17] Zehnder M, Kosicki D, Luder H, Sener B, Waltimo T. Tissuedissolving capacity and antibacterial effect of buffered and unbuffered hypochlorite solutions. Oral Surg Oral Med Oral Pathol Oral Radiol Endod 2002;94:756-762.

[18] Andrabi SM, Kumar A, Kumar Tewari R, Kumar Mishra S, Iftekhar $\mathrm{H}$. An in vitro SEM study on the effectiveness of smear layer removal of four different irrigations. Iran Endod J 2012;7:171-176.

[19] Yamada RS, Armas A, Goldman M, Lin PS. A scanning electron microscopic comparison of a high volume final flush with several irrigating solutions: Part 3. J Endod 1983;9:137-142.

[20] Ashraf H, Asnaashari M, Darmiani S, Birang R. Smear Layer Removal in the Apical third of root canals by two chelating agents and laser: A comparative in vitro study. Iran Endod J 2014;9:210-214.

How to cite this article: Kalyoncu IO, Giray FE, Durmus B, Garip Berker Y, Tanboga I. Efficacy of MTAD solution and Er:YAG laser in smear layer removal from extracted root canals: A SEM Evaluation. Clin Exp Health Sci 2021; 11: 9-13. DOI: 10.33808/ clinexphealthsci.794726 